\title{
Gastrointestinal motility in people with type 1 diabetes and peripheral neuropathy. Reply to Marathe CS, Rayner CK, Jones KL, et al [letter]
}

\author{
Adam D. Farmer ${ }^{1,2,3}$ • Anne Grave Pedersen ${ }^{3,4}$ - Birgitte Brock ${ }^{4,5}$. \\ Poul Erik Jakobsen ${ }^{6}$ - Jesper Karmisholt ${ }^{6}$ - Sahar D. Mohammed ${ }^{2}$ - S. Mark Scott ${ }^{2}$. \\ Asbjørn Mohr Drewes ${ }^{3}$ - Christina Brock ${ }^{3,5,7}$
}

Received: 29 June 2017 / Accepted: 3 July 2017 /Published online: 17 September 2017

(C) Springer-Verlag GmbH Germany 2017

Keywords Diabetes mellitus · Gastrointestinal dysfunction · Gastrointestinal transit · Pan-enteric dysfunction · SmartPill

\section{Abbreviation \\ WMC Wireless motility capsule}

To the Editor: We would like to thank Marathe and colleagues for their interest in our recent paper concerning the pan-enteric prolongation of gastrointestinal transit times and an altered caecal $\mathrm{pH}$ profile in people with type 1 diabetes and peripheral neuropathy $[1,2]$. In their letter, they raise a number of salient and important issues regarding the interpretation of our data.

Christina Brock

christina.brock@rn.dk

1 Department of Gastroenterology, University Hospitals of North Midlands, Stoke on Trent, UK

2 Centre for Neuroscience and Trauma, Blizard Institute, Wingate Institute of Neurogastroenterology, Barts and the London School of Medicine and Dentistry, Queen Mary University of London, London, UK

3 Mech-Sense, Department of Gastroenterology and Hepatology, Aalborg University Hospital, Mølleparkvej 4, DK-9000 Aalborg, Denmark

4 Clinical Biochemistry, Aarhus University Hospital, Aarhus, Denmark

5 Department of Clinical Medicine, Aarhus University, Aarhus, Denmark

6 Department of Endocrinology, Aalborg University Hospital, Aalborg, Denmark

7 Department of Drug Design and Pharmacology, University of Copenhagen, Copenhagen, Denmark
As Marathe et al point out, our study is subject to a number of limitations [2]. The major limitation is that the study is an observational secondary analysis of an ongoing clinical trial and, thus, not designed to address causality between exposure and outcome. However, emerging evidence from other studies provides a plausible potential causal link between pan-enteric prolongation of transit times and diabetic sensory peripheral neuropathy, owing to a shared pathogenesis [3, 4]. For instance, chronic hyperglycaemia directly leads to neuronal impairment, with subsequent indirect consequences that include atherosclerosis compromising neuronal blood flow, neuro-inflammation, heightened oxidative stress and immunological activation of e.g. glia cells. In conjunction, these systemic alterations may result in pathological alterations in, not only peripheral sensorimotor nerves, but also the autonomic nervous system, a branch of which is the enteric nervous system [5]. We readily acknowledge that future research should focus on the characterisation of these aspects in those individuals with co-existing diabetic sensorimotor and autonomic neuropathy.

We agree that it would be desirable to compare transit times in people with diabetes, encompassing those with and without a peripheral neuropathy, and in both type 1 and type 2 diabetes. We are currently undertaking a study to address these outstanding questions. However, we would still argue that our data supports the conclusions that gastrointestinal dysmotility is present in a proportion of people with type 1 diabetes with neuropathy, as demonstrated using the wireless motility capsule (WMC).

We would concur that previous studies examining gastric emptying demonstrate a wide variety of results in people with diabetes and, furthermore, that comparison between studies is challenging because of differences in demographics, clinical factors and methodology. We agree that the WMC has not been validated in people with type 1 diabetes and peripheral neuropathy per se but, likewise, a similar argument could be 
made regarding scintigraphy, as limited data exist. Whilst gastric scintigraphy is considered the gold standard, considerable variations in practice remain despite international efforts for standardisation [6]. Whilst Marathe et al highlight the inherent advantages of scintigraphy [2], it must be noted that this investigation involves a dose of ionising radiation, albeit small. However, in contrast to gastric scintigraphy, the WMC allows measurement of multi-segmental transit times in a single test, which is time and resource intensive when using whole gut scintigraphy [7]. Furthermore, the WMC also delineates pressure and contractility variables that may be salient to gastrointestinal symptoms in diabetes, although further work is needed in this regard $[8,9]$. Despite it being well established that severe acute and chronic hyperglycaemia can retard gastric emptying, evidence remains limited that such delayed gastric emptying is an independent risk factor for impairment of glycaemic control [10]. In our dataset, we did not find any statistically significant correlations between blood glucose immediately prior to ingestion of the WMC, or glycaemic index, and gastric emptying time. Our data agree with the comment regarding the lack of concordance in single segmental transit delay with other segments [2], although a small proportion of individuals have pan-enteric prolongation of transit times. Whilst our data has potential implications for clinical care of patients with type 1 diabetes and neuropathy, our study does not provide evidence for causality, which warrants further examination in future studies investigating pharmacological glucose-regulating interventions; nor does it provide evidence for the underlying pathophysiology of type 1 diabetes and neuropathy, which should be investigated in future studies using glycaemic clamps.

Funding Work in the authors' lab is supported by Nordisk Scandinavia AS and the Danish Diabetes Academy founded by the Novo Nordisk Foundation. The study sponsor was not involved in the design of the study; the collection, analysis, and interpretation of data; writing the report; or the decision to submit the report for publication.
Duality of interest The authors declare that there is no duality of interest associated with this manuscript.

Contribution statement All authors were responsible for drafting the article and revising it critically for important intellectual content. All authors approved the version to be published.

\section{References}

1. Farmer AD, Pedersen AG, Brock B et al (2017) Type 1 diabetic patients with peripheral neuropathy have pan-enteric prolongation of gastrointestinal transit times and an altered caecal $\mathrm{pH}$ profile. Diabetologia 60:709-718

2. Marathe CS, Rayner CK, Jones KL, Horowitz M (2017) Gastrointestinal motility in people with type 1 diabetes and peripheral neuropathy. Diabetologia https://doi.org/10.1007/s00125-0174391-3

3. Tesfaye S, Boulton AJ, Dyck PJ et al (2010) Diabetic neuropathies: update on definitions, diagnostic criteria, estimation of severity, and treatments. Diabetes Care 33:2285-2293

4. Brock C, Softeland E, Gunterberg V et al (2013) Diabetic autonomic neuropathy affects symptom generation and brain-gut axis. Diabetes Care 36:3698-3705

5. Furness JB (2006) The enteric nervous system. Blackwell Publishing, Oxford

6. Abell TL, Camilleri M, Donohoe K et al (2008) Consensus recommendations for gastric emptying scintigraphy: a joint report of the American Neurogastroenterology and Motility Society and the Society of Nuclear Medicine. J Nucl Med Technol 36:44-54

7. Camilleri M, Bharucha AE, di Lorenzo C et al (2008) American Neurogastroenterology and Motility Society consensus statement on intraluminal measurement of gastrointestinal and colonic motility in clinical practice. Neurogastroenterol Motil 20:1269-1282

8. Barshop K, Staller K, Semler J, Kuo B (2015) Duodenal rather than antral motility contractile parameters correlate with symptom severity in gastroparesis patients. Neurogastroenterol Motil 27:339-346

9. Barshop K, Kuo B (2015) Connecting the dots between gastrointestinal motility and symptoms using wireless motility capsule testing. Dig Dis Sci 60:1120-1122

10. Halland M, Bharucha AE (2016) Relationship between control of glycemia and gastric emptying disturbances in diabetes mellitus. Clin Gastroenterol Hepatol 14:929-936 